\title{
REVIEWS.
}

\section{A PRACTICAL MANUAL OF DISEASES OF THE CHEST.}

\author{
By Maurice Davidson. Oxford University \\ Press. 1935. \\ Price $42 /$ -
}

In spite of the fact that diseases of the chest form such a considerable portion of the work of the family doctor, it is remarkable that there should be so few moderately sized yet thorough treatises on the subject. True, there are several well-known monographs of varying magnitude devoted to certain special fields, but there was a real need for a comprehensive work embracing the whole subject of diseases of the respiratory system. It is, therefore, with pleasure that we welcome the Manual by Dr. Maurice Davidson as it exactly fills this gap.

This is a volume born of ripe and extensive experience and the views are expressed in a terse and judicious manner and couched in a language which is never ambiguous. Naturally, the clinical manifestations receive most attention and the general descriptions of these are excellent and are enriched by records of many illustrative cases. Pathology and Epidemiology, however, are by no means neglected. And last but not least, one must remark on the fulness and sanity of the sections dealing with treatment which always include a discussion of the most recent additions which bacteriology and surgery have made possible. Special mention must also be made of the excellent reproductions of the numerous drawings of naked-eye appearances and X-ray pictures which, almost without exception, reveal the desired features.

It is, of course, always possible to find some points for criticism. On the question of broncho-pneumonia the important distinction between the primary and secondary forms is more or less neglected and we were astonished to notice no mention of Southey's tubes which provide such a safe and easy method of performing paracentesis thoracis. We also regret that Dr. Davidson did not express any opinions on the question of the pulmonary manifestations of the rheumatic infection. But such criticisms do not in the least detract from the value of a most excellent text-book which can be recommended with the greatest confidence.

\section{OPERATIVE SURGERY.}

By C. R. Whittaker.

Price $4 / 6$.

This book is one of the Catechism Series; a clearly printed and well-bound small volume of two hundred and twenty-three pages, which fits comfortably into the student's pocket.

The surgical anatomy is well described, the line diagrams are good and adequately labelled ; all students should easily be able to reproduce these useful sketches in an examination. More of them would be welcome.

The book is probably intended for the undergraduate preparing for examination. From its size and title one would expect careful discrimination to have been exercised in the choice of the operations discussed, and from the fact that it is the fifth edition, one anticipated masterly yet brief descriptions of the essentials-it was rather disappointing.

One hoped that only proved operations of current practice would be included, but fouro operations for saphena varix, two for? splenopexy, and Kennedy's and Sever's operation for Erb Duchenne's paralysis are given. These are seldom performed now, and are, therefore, unlikely to be asked for at examination.

On page seventy-two is an error: the " metatarsal phalangeal" joint being inserted instead of the "tarso-metatarsal" joint.

The average student would have difficulty in performing Symes amputation, a classical and occasionally performed operation, from the directions in the book.

In the exposure of the humerus, and of the femur, the incisions described by Henry are probably better than the older approaches given here; at any rate they are worthy of inclusion as alternatives. In fractures of the neck of the femur, an eightinch steel nail is no longer used, but a Smith Petersen stainless steel flanged pin is inserted.

Many operations described are either historical or are no longer practised, whilst established procedures such as the radical 